\title{
Diseño y Elaboración del Texto de Inmunología Básica y su correlación clínica
}

\section{Design and Development of the Basic Immunology Text and its clinical correlation.}

DOI: $10.46932 /$ sfjdv2n2-027

Received in: january 1st, 2020

Accepted in: March 30th, 2020

\section{Dr. Ángel José Chú Lee}

Mg Sc. Especialista en Medicina Interna - Acupuntura China

Profesor Titular Inmunología Universidad Técnica de Machala. Autor Texto Inmunología Básica y Clínica - Año 2015 - UTMACH

E-mail: achu@utmachala.edu.ec

\section{Dra. Carina Alexandra Serpa Andrade}

Mg Sc. Magister en Medicina Forense- Universidad de Guayaquil

Profesora Inmunología Universidad Técnica de Machala

E-mail: cserpa@utmachala.edu.ec

\section{Dra. Sylvana Alexandra Cuenca Buele}

Especialista en Anatomía Patológica - Universidad del Azuay

Profesora Titular Universidad Técnica de Machala

Autora Texto Inmunología Básica y Clínica - Año 2015 - UTMACH

E-mail: sacuenca@utmachala.edu.ec

\section{Lcda. Lina Maribel Barreto Huilcapi}

Licenciada en Ciencias de la Educación Especialización Lengua Inglesa - Universidad de Cuenca.

Profesora Universidad Técnica de Machala.

Autora Texto Inmunología Básica y Clínica - Año 2015 - UTMACH

E-mail: lbarreto@utmachala.edu.ec

Srta. Meiling Paulette Chú Lee Riofrio

Estudiante 5to Semestre - Facultad de Medicina

Universidad de Cuenca.

Co autora Texto de Inmunología Básica y Clínica - 2015 - UTMACH

E-mail: meiling51012@gmail.com

Dra. Ximena Damaris Maldonado Riofrio

Medico General - Universidad Técnica de Machala

Co autora Texto de Inmunología Básica y Clínica - 2015 - UTMACH

E-mail: xdamarisr@gmail.com

Srta. Barbara Fernanda Verdaguer Granda

Estudiante 4to. Semestre Carrera de Medicina

Universidad Técnica de Machala

E-mail: bverdaguer1@utmachala.edu.ec

Srta. Josselyn Ariana Cabrera Honores

Estudiante 4to. Semestre Carrera de Medicina 
Universidad Técnica de Machala

E-mail: jcabrera19@utmachala.edu.ec

\section{RESUMEN}

Objetivo: Elaboración Texto de Inmunología Básica y Clínica.

Métodos: Recolección, revisión de la Bibliografía de los últimos cinco años con la información más actualizada de la Inmunología para luego con un editor plasmarlo en Texto de Inmunología Básica y Clínica.

Resultados: El Texto elaborado de Inmunología Básica y Clínica estructurado con sus 4 Unidades basado en los contenidos del Syllabus de Inmunología.

Conclusiones: El texto de Inmunología Básica y Clínica servirá como Texto guía en la asignatura de Inmunología que se imparte en el Cuarto semestre de la Carrera de Medicina, malla rediseñada.

Palabras clave: Inmunología, Texto guía, Elaboración Texto.

\section{ABSTRACT}

Objective: Elaboration of Basic and Clinical Immunology Text.

Methods: Collection and review of the Bibliography of the last five years with the most updated information on Immunology and then with an editor to translate it into a Text of Basic and Clinical Immunology.

Results: The elaborated Text of Basic and Clinical Immunology structured with its 4 Units based on the contents of the Immunology Syllabus.

Conclusions: The text of Basic and Clinical Immunology will serve as a guide text in the subject of Immunology taught in the fourth semester of the Medicine Career, redesigned syllabus.

Key words: Immunology, Guide text, Text development.

\section{INTRODUCCIÓN}

Hablar de inmunología es hablar del Sistema Inmune que protege a nuestro organismo a través de tres propiedades principales a) Capacidad de reconocer lo propio y oponerse a lo extraño b) Especificidad, y c) Memoria Inmunológica.

Haciendo un vistazo a la Historia de la Humanidad, vemos que desde La China Milenario 2000 A.C, se empezó la VARIOLIZACION, luego en 1796, Edward Jenner llevó más lejos su experimento al inyectar un fluido extraído de una llaga de viruela de vaca de una sirvienta en un niño de ocho años, Jenner es considerado Padre de la Inmunología Moderna, llegando hasta la década de los 80 en donde aparece el V.I.H. en individuos enfermos sin respuesta Inmunitaria. Y es aquí, que la Inmunología siempre considerado como un Capitulo de la Biología se despega y se convierte en una asignatura nueva e independiente que se dictan en el pensum de estudios de la mayoría de las Carreras de Ciencias Médicas - Medicina a nivel Mundial.

La inmunología ha tenido una transformación constante desde que se iniciaron los primeros estudios a través de la inoculación de fluidos provenientes de una pústula de la viruela en un niño en el 
siglo XVIII originando inmunidad. Ya en el siglo XIX el profesor Luis Pasteur obtuvo la primera vacuna de microorganismos vivos. Melchnikov en 1883 postula la teoría de los fagocitos creando las bases iniciales teóricas de la inmunidad celular y Emil Van Bering en 1890 demuestra la presencia de antitoxinas en el suero capaces de defender de enfermedades introduciendo los primeros postulados de la inmunología humoral. Kraus en 1897 visualiza por primera vez la reacción antígeno-anticuerpo. Además aparece la definición de "tolerancia inmunológica" ha evolucionado lentamente después de la observación de R.D. Owen (1897) en terneros gemelos dicigóticos, en los que se observaba un intercambio constante de sangre embrionaria, que volvía tolerantes a los antígenos tisulares de ambos animales entre sí.

En 1902 se describe por Karl Landsteiner la reacción de aglutinación del sistema de antígenos naturales en eritrocitos humanos. A pesar de haberse realizado con éxito transfusiones de sangre y de tejidos de la medula ósea, no es hasta el descubrimiento del Sistema Principal de Histocompatibilidad (HLA), que permite un desarrollo acelerado de la trasplantología. La aparición de anticuerpos monoclonales en la década de 1970 abrió una ventana para el de enfermedades como el cáncer y enfermedades crónicas no transmisibles, lo que le permitió la obtención del premio nobel de medicina a los doctores Niels K. Jener, George J. F. Köhler y César Milstein.

Hasta la presente fecha, Todas las enfermedades que tenía una etiología desconocida, idiopática, etc. se lo encuentra inmerso en el campo de la Inmunología y el empleo de las Terapias Biológicas en la Enfermedades Autoinmunes es actualmente una gran realidad.

A lo largo de la historia, los seres humanos han conseguido desarrollar vacunas para algunas enfermedades potencialmente mortales, entre ellas la meningitis, el tétanos, el sarampión y la poliomielitis, etc. Las vacunas contienen partes atenuadas o inactivadas de un organismo específico (antígeno) que provoca una respuesta inmunitaria en el cuerpo. Las vacunas más recientes contienen las 'instrucciones' para producir antígenos, en lugar del antígeno en sí mismo. Independientemente de que la vacuna contenga el antígeno o las instrucciones para que el cuerpo lo produzca, esa versión atenuada no provocará la enfermedad en la persona vacunada, pero inducirá al sistema inmunitario a responder como lo hubiese hecho en su primera reacción ante el patógeno real.

Algunas vacunas requieren la administración de múltiples dosis a intervalos de semanas o meses. En ocasiones, esto es necesario para posibilitar la producción de anticuerpos de larga vida y el desarrollo de células de memoria. De esa forma, el cuerpo se prepara para combatir el organismo específico causante de la enfermedad y recordar el patógeno para combatirlo rápidamente si ello fuera preciso en el futuro.

Desde 11 marzo del 2020, la Organización Mundial de la Salud (OMS) decreta PANDEMIA COVID 19 a consecuencia de un Virus que ingresan a las Vías Respiratorias produciendo una 
TORMENTA DE CITOCINAS. Debido a esta Emergencia Sanitaria estamos confinado en nuestros hogares debido a la Segunda Oleada del COVID 19 que están afectando a todos los países del Mundo, incluyendo nuestro lindo Ecuador.

Cada día hay nuevos estudios en relación al COVID 19 que implica compromiso del Sistema Inmunológico. Por la cual desarrollaron diferentes ensayos de vacunas. Desde diciembre 2020 se iniciaron a inmunizar a la población con la Vacuna PFIZER, la cual son dos dosis administradas con un intervalo de 3 semanas (21 días), quien ha demostrado tener los mejores resultados. Aunque existen otras vacunas entre las cuales tenemos: Janssen de Johnson \& Johnson, Sinovac china, Iniciativa covax facility (pool de vacunas), Novavax/serum institute (india), Oxford/Astrazeneca, Gamaleya center (sociedad gestora del fondo ruso de inversión directa), entre otras.

Con el diseño y elaboración del Texto Inmunología Básica y Clínica actualizamos y mejoramos el nivel académico en la asignatura de Inmunología mediante una propuesta pedagógica realizado por una iniciativa del grupo selectos de 14 estudiantes del 4to semestre Paralelo A II Promoción de la Malla Rediseño, quienes se propusieron investigar, descargar y plasmar los conocimientos más elementales y actualizados que deberían conocer todos los estudiantes de Pregrado de la Carrera de Medicina.

\section{OBJETIVOS}

\subsection{OBJETIVO GENERAL}

- Determinar la importancia de un texto guía para la enseñanza de la asignatura Inmunología para los estudiantes de la carrera de Medicina de la Universidad Técnica de Machala.

\subsection{OBJETIVOS ESPECÍFICOS}

- Señalar la importancia de la asignatura de Inmunología que se imparte en el Cuarto Semestre, Malla del Rediseño en la carrera de Medicina de la Universidad Técnica de Machala.

- Justificar la necesidad de un texto guía para el aprendizaje de la asignatura de Inmunología en la carrera de medicina.

Durante el proceso en el diseño y elaboración del texto de Inmunología Básica y Clínica, encontramos trabajos realizados por diversos autores que han publicados sobre las diferentes modalidades para mejorar el proceso de enseñanza aprendizaje en la asignatura de Inmunología: 
En este proyecto se propone el desarrollo de un informe avanzado de prácticas realizado conjuntamente por grupos de unos 20 alumnos aproximadamente, sobre cada una de las prácticas que se imparten en la asignatura de Inmunología General del Grado de Biología. Los informes contuvieron una introducción, unos objetivos, unos materiales y métodos, unos resultados, una discusión, así como las respectivas conclusiones y la bibliografía utilizada; quedando patente todos aquellos integrantes del grupo que hubiesen trabajado activamente en la elaboración del mismo. El plazo para elaborar el material fue de dos semanas tras finalizar la respectiva práctica, pasado el cual, fueron entregados a los profesores en formato PDF, con el objetivo de ser corregidos y evaluados. Una vez finalizadas todas y cada una de las prácticas, se adjuntaron las memorias, formando un único manual de referencia que les sirvió a los alumnos para estudiar la parte práctica de la asignatura. Asimismo, con la elaboración de este manual se fomentó el trabajo colaborativo entre los estudiantes, vital para su futura vida laboral. (Sempere Ortells, 2017)

En cambio, en este proyecto supone una continuación de la red 3830 de la convocatoria 2016/2017. Para esta actividad, los 158 alumnos matriculados en Inmunología General se dividieron en 7 grupos de prácticas y realizaron un informe de una de las clases que les fue asignada por los profesores responsables de la asignatura. Los informes se realizaron con la herramienta Wiki del Moodle de UA Cloud y se escribieron tanto en castellano (6 informes), como en inglés (1 informe). Tras su corrección, se observó que las calificaciones obtenidas en los distintos apartados, como en la calidad del contenido, la ortografía, coherencia y cohesión, la calidad de las imágenes y la calidad de la maquetación fueron peores que para los informes controles y colaborativos, realizados durante el curso 2016/2017. Además, al analizar el porcentaje de plagio en los siete informes de prácticas se observaron valores elevados que alcanzaron hasta el 38\%. Estas bajas calificaciones también se observaron en las notas finales de la asignatura, siendo menores que las de los alumnos matriculados durante el curso 2016/2017. Estos hechos podrían explicarse por un desconocimiento de los estudiantes en el manejo de la herramienta Wiki, ya que fue la primera vez que trabajaron con ella. (Martínez Peinado, P, 2018)

Inmunología General supone una materia compleja debido a los numerosos conceptos nuevos que deben aprender los alumnos. Debido a esto y al auge de las TIC, se ha creado un perfil del Grupo de Inmunología en Facebook y Twitter con el usuario@ inmunologiaUA donde los profesores responsables de la asignatura publican noticias actuales o descubrimientos novedosos sobre cáncer, inmunodeficiencias, nuevos tratamientos, etc. De este modo, se pretende motivar a los estudiantes para que se den cuenta de la aplicabilidad de las nociones impartidas en clase. Además, se les propuso que fueran ellos los que publicaran noticias, 
mediante el hashtag\# inmuno UA para favorecer su involucración en la asignatura. Aunque sólo 8 alumnos publicaron 11 noticias, en la encuesta final que respondieron de forma anónima, la mayoría de los estudiantes consideró que las publicaciones en las redes sociales les ayudaron a prepararse la asignatura de cara a los exámenes finales. (García, 2018)

En la asignatura de Inmunología General, que se imparte en el tercer curso del Grado en Biología, han decidido por los nuevos métodos de enseñanza, como el aprendizaje basado en problemas (ABP) y el m-learning, En esta actividad, el profesor explicó un caso clínico que consistía en una niña que sufría Síndrome de Sjögren. A través de ocho preguntas que les fue formulando, los alumnos, reunidos en grupos de cuatro o cinco personas, debieron buscar información sobre síntomas o técnicas diagnósticas. Después tuvieron que escribir en la aplicación de Socrative ${ }^{\circledR}$ la respuesta a cada pregunta, para que todos los estudiantes pudieran verla y el profesor pudiera explicar la contestación correcta. Cuando terminó esta actividad, se realizó una encuesta de valoración anónima y la mayoría de los estudiantes estuvieron de acuerdo en que la actividad les había ayudado a asentar los conocimientos impartidos en clase, así como que el uso de Socrative ${ }^{\circ}$ había dinamizado la realización del caso clínico y se había fomentado el trabajo colaborativo. Finalmente, el alumnado que realizó esta actividad obtuvo una mayor calificación en el examen final de la asignatura, lo que ratifica nuestra hipótesis de que el ABP y el m-learning fomentan el autoaprendizaje. (Martínez Peinado, 2019)

\section{METODOLOGÍA}

Es un estudio descriptivo basado en la recopilación de información cinética de los últimos cinco años de bases de datos de revistas indexadas como Scielo, Pubmed, Dialnet. Web de Ciencies. Además de textos de referencia pertinentes actualizado del área de estudio.

\section{RESULTADOS}

El texto de inmunología Básica y Clínica consta de cuatro unidades con actividades de refuerzo de contenidos e imágenes representativas con el fin de dotar de información reciente y clara para el estudio de la Inmunología.

UNIDAD I. Generalidades e Historia de la Inmunología.

UNIDAD II. Inmunología Básica. Estudio de los mecanismos involucrados en la respuesta inmunitaria. Órganos linfoides primarios y secundarios.

UNIDAD III. Inmunología Clínica. Análisis de las enfermedades que provocan los trastornos del sistema inmunológico.

UNIDAD IV. Terapias Inmunitarias. Estrategias de tratamiento para estimular o reponer el sistema 
inmunitario.

\section{DISCUSIÓN}

La educación médica en el siglo XXI exige de un estudiante capaz de captar las conexiones entre las diferentes asignaturas para dar solución a determinada problemática y puedan integrar aquello que se les enseñó aisladamente en las asignaturas. (Lage, 2018)

Para la planificación del curso, su desarrollo de la enseñanza, se han considerado la naturaleza del conocimiento relativo al tema Inmunología, además de la búsqueda activa de información en diferentes bibliografías, el contexto de la enseñanza y la necesaria relación de dicho conocimiento con las necesidades del alumnado para comprender su realidad e intervenir en ella. La práctica docente basada en la integración entre los conocimientos específicos y los conocimientos pedagógicos, mediante la realización del libro, ha contribuido para que los alumnos participaran activamente en las clases del curso y para que extendieran los conocimientos sobre la dinámica del sistema inmune a situaciones cotidianas variadas.

El sistema inmunitario funciona con complejas interacciones celulares que le permiten "decidir" cuando reaccionar con una respuesta inmune efectora y cuando con una respuesta tolerante. Tratar de entender cómo trabajan estos mecanismos ha sido siempre el problema fundamental de la inmunología. A inicios del pasado siglo numerosas teorías han sido enunciadas, cada una con aciertos y desaciertos; sin embargo, han colaborado con un granito de arena a nuestros conocimientos modernos. Actualmente, se sabe que para que ocurra una respuesta efectora es necesario la existencia de señales de peligro y daño tisular, acompañado de un antígeno con propiedades inmunogénicas, que debe ser reconocido por un clon específico de linfocitos el cual se expande y se obtienen células hijas con igual especificidad, pero con afinidad mayor que las células vírgenes. Adicionalmente, que en periferia existen clones autorreactivo los cuales tienen un papel fisiológico y son constantemente regulados por mecanismos de tolerancia periférica para evitar el desarrollo de enfermedades autoinmunes. El sistema inmune es complejo y diverso tanto como es la diversidad de antígeno al que se expone; es por eso que llegar a comprenderlo del todo es un reto al entendimiento humano. (Alonso Remedios, Alaín , 2016)

Según Serrano Barrera, Orlando y colaboradores, (Serrano Barrera, 2005) La Inmunología no solo aporta conocimientos indispensables en la comprensión de la interacción hombre-ambiente, en lo relativo a la dicotomía resistencia versus susceptibilidad a infecciones bajo el supuesto de que toda enfermedad infecciosa es una enfermedad del sistema inmune

De acuerdo a Abreu et al (Abreu de Andrade, 2014) el uso del organizador previo en la enseñanza de la Inmunología ha mostrado potencialmente significativo para el aprendizaje, pues ha permitido el 
establecimiento de un puente entre lo que el alumnado ya sabía y lo que era relevante (la idea central homeostasis) para el aprendizaje de Inmunología Básica.

La motivación y el interés son críticos en el aprendizaje y el rendimiento académico. Los alumnos prestan más atención en una asignatura en particular cuando se retiene su interés, desarrollando sus dotes de observación, memorización, comprensión y pensamiento crítico, todos ellos herramientas imprescindibles en la investigación científica. Por tanto, se hace evidente la necesidad de nuevas estrategias y herramientas para la docencia de asignaturas de Inmunología. (Torres-Gomez*, 2918)

\section{CONCLUSIONES}

El diseño y la elaboración del Texto de Inmunología Básica y Clínica permitirá:

- Provee información comprensiva sobre el sistema inmune.

- Los temas son secuenciales, se desarrollan desde lo elemental hasta lo complejo.

- Presenta imágenes representativas que facilitan el aprendizaje de los conocimientos.

- En definitiva, la implementación de un texto guía para el aprendizaje de la Inmunología, facilitaría cumplir con los objetivos y competencias curriculares de la asignatura, mejoraría la metodología de investigación al indagar en fuentes actualizadas y al ser enfocado a las necesidades didácticas de las ciencias de la salud mejoraría paralelamente el desempeño académico en las materias de especialidad.

- El Texto se encuentra redactado en un lenguaje muy amigable para tener una lectura amena y comprensible de la misma. Se lo dividió en 4 Unidades: Historia y Generalidades de la Inmunología, Inmunología Básica, Inmunología Clínica y Terapia Inmunitaria.

- El presente texto servirá como Texto Guía en la asignatura de Inmunología y habrá cumplido sus objetivos cuando sea utilizado por estudiosos de la Inmunología, incluyendo a los estudiantes de la Carrera de Medicina, Universidad Técnica de Machala. 


\section{REFERENCIAS BIBLIOGRÁFICAS:}

1. Abreu de Andrade, V., Maximo Pereira, M., Cremonini de Araújo-Jorge, T., \& Coutinho Silva, R. (2014). El uso de un organizador previo en la enseñanza de Inmunología. Revista Eureka Sobre Enseñanza $Y$ Divulgación De Las Ciencias, 12(1), pp. 38-54. Recuperado a partir de https://revistas.uca.es/index.php/eureka/article/view/2901

2. Alonso Remedios, Alaín y Pardo Martínez, Daynelis y Zabala Enrique, Bárbara Teresa y Barrueta Tirado, Servilio y Albelo Amor, Omaida (2016). Evolución del pensamiento en Inmunología. MediSur, 14 (2), 204-212. [Fecha de Consulta 25 de Diciembre de 2020]. ISSN:. Disponible en: https://www.redalyc.org/articulo.oa?id=1800/180045580015

3. Berlanga, Carlos (2016). Reseña del libro "Inmunología molecular, celular y traslacional". Lenin Pavón Romero, María C. Jiménez Martínez, María Eugenia Garcés Álvarez Editorial Wolters Kluwer, Barcelona, 2016. Salud Mental, 39(6),323-324.[fecha de Consulta 28 de Diciembre de 2020]. ISSN: 01853325. Disponible en: https://www.redalyc.org/articulo.oa?id=582/58248800007

4. Cuenca Buele, Sylvana Alexandra et al. (2018); Introducción y clasificación actual de las enfermedades autoinmune. Colección REDES 2018 - Capítulos - ISBN : 978-9942-24-121-4 Disponible: http://repositorio.utmachala.edu.ec/handle/48000/14198

5. Chu Lee, Ángel José et al. (2015). Inmunología básica y clínica. Ecuador: Universidad Técnica de Machala. Disponible :http://repositorio.utmachala.edu.ec/handle/48000/6650

6. Lage, Miladys Ramos, Vidal Rojo, Carmen, Conde Fernández, Berto Delis, \& Pérez García, Lizandro Michel. (2018). Propuesta de procedimientos para elaborar tareas integradoras en asignaturas de la carrera Medicina. Gaceta Médica Espirituana, 20(3), 101-111. Recuperado en 25 de diciembre de 2020, de $89212018000300101 \& \operatorname{lng}=$ es\&tlng=es.

7. Martínez Peinado, P., et al. (2018) "Creación de una enciclopedia (Wiki) de los contenidos y conceptos explicados en las prácticas de Inmunología General del Grado en Biología, mediante la aplicación Moodle de UA Cloud". En: Roig-Vila, Rosabel (coord.). Memorias del Programa de RedesI3CE de calidad, innovación e investigación en docencia universitaria. Convocatoria 2017-18= Memòries del Programa de Xarxes-I3CE de qualitat, innovació i investigació en docència universitària. Convocatòria 2017-18. Alicante: Universidad de Alicante, Instituto de Ciencias de la Educación (ICE). ISBN 978-84-09-07041-1, pp. 3057-307

8. Martínez Peinado, P., et al. (2019) "Aprendizaje de la Inmunología a través de casos clínicos mediante el uso de Socrative ${ }^{\circledR}$ ". En: Roig-Vila, Rosabel (coord.). Memòries del Programa de XarxesI3CE de qualitat, innovació i investigació en docència universitària. Convocatòria 2018-19= Memorias del Programa de Redes-I3CE de calidad, innovación e investigación en docencia universitaria. Convocatoria 2018-19. Alacant: Institut de Ciències de l'Educació (ICE) de la Universitat d'Alacant. ISBN 978-84-09-15746-4, pp. 2461-2475

9. Pavón Romero, Lenin; Jiménez Martínez, María C.; Garcés Álvarez, María Eugenia (2016). Inmunologia Molecular, Celular y Traslacional. Editorial Wolters Kluwer. Edición lra.

10. Rojas W, Anaya Cabrera J, Aristizabal B B, Cano R L, Gómez O L, Lopera HD (2015). Inmunologia de Rojas. 17th ed. Colombia: Corporación para Investigaciones Biológicas (CIB). 
Disponible en: (99+) (PDF) Inmunologia de Rojas 17e 20190612116259 6i7bfq | Carolina Hernandez Academia.edu

11. Serrano Barrera, Orlando R., Robinson Rodríguez, Rosa J., Castellanos Martínez, Rosa, \& Guevara Rosales, Mercedes. (2005). La Inmunología en la formación de pregrado de la docencia médica. Educación Médica Superior, 19(4) Recuperado en 25 de diciembre de 2020, de http://scielo.sld.cu/scielo.php?script=sci_arttext\&pid=S0864-21412005000400003\&lng=es\&tlng=es.

12. WHO SAGE roadmap for prioritizing uses of COVID-19 vaccines in the context of limited supply. Geneva: World Health Organization; 2020 , link (https://www.who.int/publications/m/item/who-WHOroadmap-for-prioritizing-uses-ofcovid-19-vaccines-in-the-context-of-limited-supply accessed 30 December 2020).

13. Novak, J., \& Lehuen, A. (2011). Mechanism of regulation of autoimmunity by iNKT cells. Cytokine, 53(3), 263-270. https://doi.org/10.1016/j.cyto.2010.11.001

14. Sakaguchi, S., Yamaguchi, T., Nomura, T., \& Ono, M. (2008). Células T reguladoras y tolerancia inmunitaria, 133(5), 775-787. https://doi.org/10.1016/j.cell.2008.05.009

15. Cañarte Alcívar, J., Anzules Guerra, J., Uscocovich Alcívar, A., Bravo Mendoza, M., Zambrano Mera, S., Bello Mendoza, V., \& Vazquez, Y. (2018). Importancia de la inmunología como ciencia. Ciencia Digital, 2(3), 28-49. https://doi.org/10.33262/cienciadigital.v2i3.135

16. Sempere Ortells, JM, eta 1. (2017) "Desarrollo de un manual de prácticas por los alumnos de Inmunología General como material de estudio de referencia, y como fomento del trabajo colaborativo". En: Roig-Vila, Rosabel (coord.). Memorias del Programa de Redes-I3CE de calidad, innovación e investigación en docencia universitaria. Convocatoria 2016-17 = Memòries del Programa de Xarxes-I3CE de qualitat, innovació i investigació en docència universitària. Convocatòria 2016-2017. Alicante: Universidad de Alicante, Instituto de Ciencias de la Educación (ICE). ISBN 978-84-697-6536-4, pp. 1697-1708

17. SP García, PM Peinado, ABL Jaén, FJN Blasco...(2018). La Inmunología en el entorno de las redes sociales (Facebook, Twitter, Paper. li) como forma de divulgación, aprendizaje y refuerzo de los conceptos estudiados en clase. Memorias del Programa de Redes-I3CE de calidad ..., Disponible en: https://scholar.google.com/citations?user=dkPIEhQAAAAJ\&hl=es\#d=gs_md_cita$\mathrm{d} \& \mathrm{u}=\% 2 \mathrm{Fcitations} \% 3 \mathrm{Fview}$ _op\%3Dview_citation\%26hl\%3Des\%26user\%3DdkPIEhQAAAAJ\%26cita tion_for_view\%3DdkPIEhQAAAAJ\%3AmVmsd5A6BfQC\%26tzom\%3D300 